\title{
Recomendaciones para la identificación con bajo costo, de Streptococcus grupo anginosus
}

\author{
COMITÉ DE MICROBIOLOGÍA CLÍNICA
}

Recommendations for diagnosis at low cost, of Streptococcus group anginosus

Streptococcus grupo anginosus antiguamente denominado grupo "milleri" es causa reconocida de infecciones clínicas. En su Comentario en este número de la revista, Elizabeth Palavecino presenta una extensa descripción de su patogenicidad $^{1}$; además se publican un Caso Clínico ${ }^{2}$ y una serie clínica ${ }^{3}$.

El Comité de Microbiología Clínica de la Sociedad Chilena de Infectología estima necesario realizar una recomendación de bajo costo para la identificación de Streptococcus grupo anginosus (Figura 1) y efectuar además una sugerencia para los laboratorios de microbiología de cómo evacuar los informes del laboratorio respecto de Streptococcus $\beta$-hemolíticos (Tabla 1), dado que existe confusión en la nomenclatura .

Los Streptococcus del grupo anginosus pueden ser $\beta$-hemolíticos y poseer antígenos de Lancefield A, C, G y F. Se pueden identificar erróneamente, si sólo se realiza test de látex para antígenos de Lancefield y no se presta suficiente atención a las características de la colonia tales como tamaño pequeño $(<0,5 \mathrm{~mm})$ y olor a caramelo. Por esta razón se propone este algoritmo de bajo costo para la identificación rutinaria en el laboratorio, aplicable también a Streptococcus no $\beta$-hemolíticos aislados de abscesos y sitios estériles, los cuales pueden corresponder al grupo anginosus. Sin embargo, en muestras estériles $S$. pyogenes y $S$. agalactiae se deberían confirmar con aglutinación con látex para grupo.

Por otro lado, si se dispone de látex para diagnóstico de grupo, aquellos Streptococcus que aglutinan con grupo $\mathrm{F}$ pueden informarse directamente como grupo anginosus y $\operatorname{los} \mathrm{C}$ y $\mathrm{G}$, bastaría con hacer la prueba de la $\beta$-D-glucuronidasa para diferenciarlos de $S$. dysgalactiae.

Tabla 1. Sugerencia para el informe de Streptococcus $\beta$-hemolíticos

\begin{tabular}{ll}
\hline Caracteríasticas microbiológicas en $\boldsymbol{\beta}$ hemolíticos & Informe Laboratorio \\
\hline Streptococcus grupo A, colonia grande PYR (+) & Streptococcus pyogenes (grupo A) \\
Streptococcus grupo B, colonia grande, CAMP(+) & Streptococcus agalactiae (grupo B) \\
Streptococcus grupo C, colonia grande & Streptococcus disgalactiae (grupo C) \\
Streptococcus grupo G, colonia grande & Streptococcus disgalactiae (grupo G) \\
Streptococcus grupo A, C, F, G, colonia pequeña, & Streptococcus grupo anginosus \\
\$-glucuronidasa (-) y Voges Proskauer $(+)$ & \\
\hline
\end{tabular}

Miembros del Comité: Stephanie Braun J, Rossanna Camponovo C, Loriana Castillo D, Erna Cona T, Béatrice Hervé E, Alejandra Fernández V, Patricia García C, Chrystal Juliet L, Mónica Lafourcade R, Olivia Trucco A, M Eugenia Pinto C. y con la participación de Paola Pidal M. Laboratorio de Microbiología, Hospital Padre Hurtado (PPM).

Recibido: 15 julio 2004

Aceptado: 15 julio 2004 


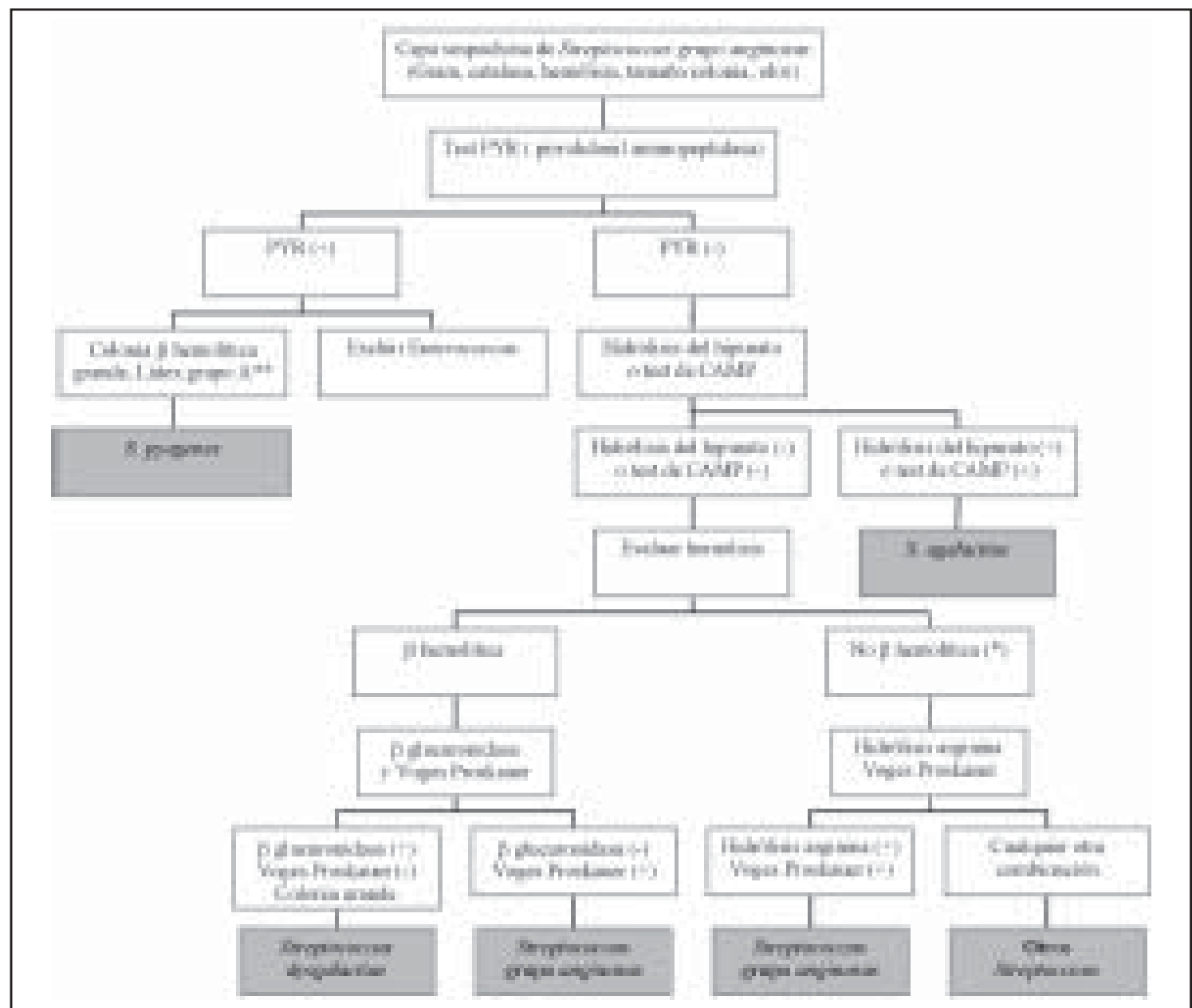

Figura 1. Algoritmo para la identificación de Streptococcus grupo anginosus.

* Se debe excluir Streptococcus pneumoniae y Streptococcus bovis.

** Cuando son muestras de líquidos estériles o hemocultivos.

Todas las reacciones hemolíticas han sido descritas en agar sangre de cordero al 5\%.

\section{Bibliografía}

1.- Palavecino E. Streptococcus grupo anginosus: ¿Es su identificación clínicamente importante? Rev Chil Infect 2004; 21: 261-7.

2.- Pidal P, Basaure J, Prado P, Alarcón P. Empiema pleural por Streptococcus grupo anginosus en un preescolar y revisión de la literatura. Rev Chil Infect 2004; 21: 248-53.

3.- Caro G, Riedel I, García P. Caracterización clínica y microbiológica de las infecciones causadas por Streptococcus grupo anginosus. Rev Chil Infect 2004; 21: 254-61.

4.- Ruoff K L, Whiley R A, Brighton D. Streptococcus.
In: Murray P R, Baron E J, Jorgensen J H, Phaller M A, Yolken R H (ed). Manual of Clinical Microbiology, $8^{\text {th }}$ ed. American Society of Microbiology, Washington DC. 2003, p 405-21.

5.- Facklam R. What happened to the Streptococci: Overview of taxonomic and nomenclature changes. Clin Microbiol Rev 2002; 15: 613-30.

6.- Hamrick H J, Mangum M E. $\beta$-hemolytic Streptococcus milleri group misidentified as Streptococcus pyogenes on throat culture. Pediatr Infect Dis J 1999; 18: 75-6.

7.- Kirby R, Ruoff K L. Cost-effective, clinically relevant method for rapid identification of $\beta$-hemolytic streptococci and enterococci. J Clin Microbiol 1995; 33: $1154-7$.

Correspondencia a:

Patricia García Cañete

E-mail:pgarcia@med.puc.cl 